Dra. Inmaculada Martín-Herrera

Universidad Pablo de Olavide. Centro Universitario San Isidoro

@ imartin@centrosanisidoro.es iD 0000-0002-8943-4766

Dr. Juan Pablo Micaletto-Belda

Universidad Pablo de Olavide. Centro Universitario San Isidoro

@ jmicaletto@centrosanisidoro.es iD 0000-0002-5424-6968
- Recibido / Received

23 de octubre de 2020

- Aceptado / Acepted 2 de noviembre de 2020

- Páginas / Pages De la 193 a la 206

- ISSN: $1885-365 \mathrm{X}$

\title{
Opiniones y actitudes de los estudiantes universitarios de Comunicación ante las fake news. Diagnóstico en un ecosistema docente
}

\section{Communication university students' opinions and attitudes towards the fake news}

\section{RESUMEN:}

El objeto de este trabajo es conocer la percepción de los estudiantes de los grados universitarios en Ciencias de la Comunicación y Comunicación Digital sobre las noticias falsas publicadas en Internet. Nos interesa conocer su postura porque como futuros informadores profesionales deberán desenvolverse en un mercado laboral marcado por esta problemática social. Se ha trabajado con una muestra dirigida para entender las actitudes en un centro universitario de Sevilla. Por su parte, la investigación responde a un diseño mixto, donde se lleva a cabo un doble estudio cuantitativo y cualitativo, para los que se han empleado sendos instrumentos de medida: un cuestionario y una entrevista estructurada con preguntas abiertas. Los resultados muestran que, en general, estos estudiantes no consumen ni comparten noticias falsas de manera periódica y que gracias a la formación adquirida saben contrastar una información. Igualmente, perciben que estos contenidos persiguen intereses políticos o económicos; y defienden la implantación de programas escolares de alfabetización mediática y digital con objeto de formar a una ciudadanía crítica con los mensajes que se difunden en la Red.

\section{PALABRAS CLAVE:}

Redes sociales; Internet; Noticias falsas; Formación universitaria.

\section{ABSTRACT:}

This paper aims to understand the relationships that exist between digital and communication Science undergraduate students and fake news on the internet. We believe it is necessary to understand the student's behaviour because they will be the future of the communication profession and also the ones who will have to deal with its significant consequences on their labor market. Data has been collected within the University of Seville in order to understand the student's attitudes. The research develops a mixed scheme approach -quantitative and qualitative analysis-, in which 
there have been used the following measuring tools: a questionnaire and an open-question interview. Results show that students dont frequently consume or share fake news because they have a foundation of knowledge that enables them to verify and filter information. They are similarly able to recognise when the published contents have other political and economic motives. For these reasons, the students support media and digital literacy programs that aim to educate the public on how to be critical towards published information on the internet.

KEY WORDS:

Social networks; Internet; Fake news; University training.

\section{Introducción}

Las noticias falsas y el periodismo representan dos conceptos contrapuestos que persisten en una sociedad digitalizada. Las fake news dificultan el derecho de los ciudadanos de acceder a una información veraz y contrastada, lo que constituye un principio básico de la actividad periodística y de los sistemas democráticos. El incremento de la difusión masiva de contenidos a través de los dispositivos móviles está generando un clima de desinformación, que afecta seriamente a la capacidad de compresión de los ciudadanos y que dibuja un nuevo paradigma laboral para las personas que gestionan la comunicación. En este contexto, se hace necesario que estos profesionales cuenten con los recursos y la formación necesaria para afrontar este fenómeno y sus posibles amenazas. Pues estos comunicadores constituyen, en sus diferentes áreas, pilares decisivos en la lucha contra la infoxicación, la desinformación, la posverdad y las fake news; conceptos que, según la literatura académica, mantendrán su impacto en la sociedad durante los próximos años. Por tanto, el objeto de este trabajo consiste en conocer la relación que los estudiantes de los grados en Ciencias de la Comunicación y Comunicación Digital mantienen con estas materias; pues serán ellos quienes constituirán la fuerza laboral de los próximos años y, por lo tanto, tendrán que hacer frente a esta problemática social. El futuro y la trayectoria de la comunicación dependerán, en gran medida, de la formación y de las competencias que estos universitarios están adquiriendo en la actualidad.

Por todo lo anterior, resulta beneficioso y de interés indagar en su actitud y en su opinión sobre diferentes aspectos relativos a las fake news. Para ello, en esta investigación se plantean los siguientes objetivos: 1) Determinar la percepción, la actitud y la preparación de estos jóvenes, como futuros profesionales de la información, ante el consumo y la gestión de este tipo de contenidos. 2) Encontrar variables relevantes, personales y académicas, que influyan en esas percepciones.

3) Conocer la relación entre dichas variables y las percepciones de los estudiantes universitarios sobre las fake news para establecer, si fuera posible, un enlace explicativo entre ellas.

\subsection{LA ERA DE LA DESINFORMACIÓN}

La sociedad está sumergida en plena revolución digital. Nunca antes en la historia el ser humano había tenido acceso a tantas fuentes de información como ahora. La ciudadanía posee cientos de opciones diferentes para el consumo de medios (cadenas de televisión, emisoras de radio, portales web, etc.). Sin embargo, es precisamente en este momento cuando la población se encuentra más desinformada (Grande, 2018); y los conceptos de infoxicación, desinformación o posverdad se relacionan entre sí (Blanco-Herrero y ArcilaCalderón, 2019) adquiriendo un significado especial en este contexto comunicativo. 
La irrupción de internet ha permitido acabar con el monopolio de la información, y ahora no sólo los editores de revistas o periódicos tienen la capacidad de emitir mensajes. Esta descentralización del poder editorial permite que los ciudadanos pueden participar en el proceso de selección, diseño y distribución de determinados contenidos como noticias, adquiriendo un rol más democrático (Hernández Serrano et al., 2017). En este contexto, las nuevas tecnologías sociales, que facilitan un rápido intercambio de información a gran escala, permiten la difusión de informaciones erróneas y engañosas (Vosoughi, Roy y Aral, 2018). Por esta razón, el consumidor de noticias falseadas también posee un papel trascendental en este fenómeno, pues la no credibilidad de determinadas informaciones es clave para frenar su propagación (Tandoc, Lim y Ling, 2017).

La sobrecarga informativa que sufren los usuarios permite hablar con fuerza del concepto de infoxicación, que ha llegado a convertirse en uno de los problemas más importantes de la era digital. Para Aguaded «asistimos, sin duda, a una hiperconexión compulsiva y una sobreinformación global, a una obsesión por la comunicación perpetua vacía de contenido» (2014: 7). A su vez, esta infoxicación es un caldo de cultivo perfecto para las fake news, definidas por el diccionario Collins (2019) como información falsa, de carácter sensacionalista, que es difundida bajo la apariencia de noticias. No se trata de un fenómeno nuevo, pero lo que sí es actual es la amplitud con la que pueden propagarse a través de internet (Fernández García, 2017). En relación a la posverdad, supone una difuminación entre la verdad y la mentira, llegándose a crear una «tercera categoría en la que el hecho que se difunde es aceptado de antemano porque encaja en nuestros esquemas mentales» (Torres, citado en Levit, 2017).

La difusión de noticias falsas ha ido en aumento, hasta el punto de que se ha convertido en un asunto urgente para toda clase de instituciones y organismos internacionales, que buscan mecanismos para combatirlas. Diversos estudios señalan que las noticias falsas se difunden más rápido en internet que las verdaderas, en especial, en las redes sociales (Martens et al., 2018), o en canales más privados como Whatsapp (Molina-Cañabate, 2019).

Respecto a sus intenciones, Khaldarova y Mervi, como se cita en Magallón-Rosa (2017), señalan que las noticias falsas «a menudo toman la forma de entretenimiento propagandístico, que es una combinación de material escandaloso, acusaciones y denuncias, música dramática e imágenes engañosas tomadas fuera de contexto» (2017: 276). De hecho, el concepto de las fake news comenzó a extenderse a nivel global durante la campaña presidencial norteamericana que enfrentó a Donald Trump y Hillary Clinton en la lucha por la Casa Blanca. También tuvo un papel significativo en el triunfo del Brexit en Reino Unido y en el rechazo del referéndum por la paz en Colombia. En todos estos casos, se hablaba de que las plataformas digitales habrían influido directamente en el transcurso de los acontecimientos, intoxicando las campañas y dando lugar a resultados inesperados marcados por el auge de los populismos y los nacionalismos (Mcnair, 2018). En esta clase de escenarios, las palabras representan una nueva arma, menos convencional, que se sitúa en el epicentro de la política, los debates públicos y los análisis académicos (Horowitz, 2019).

La Unión Europea ha puesto en marcha un plan de acción para combatir y protegerse de la desinformación y las fake news, pues considera que representa una amenaza grave, un ataque a la democracia, que afecta a la estabilidad institucional de las naciones (Comisión Europea, 2018a). A la hora de actuar en consecuencia, es necesario reivindicar el papel que los profesionales de la información tienen a la hora de formar usuarios críticos; pues esta ins- 
trucción es la mejor arma que existe ante la proliferación de las noticias falsas (López-Borrull, Vives-Gràcia y Badell, 2018). Existen bulos nacidos en Internet que han llegado a publicarse en los medios tradicionales. Con la intención de evitar una incorrecta interpretación de la realidad o el desprestigio ligado a la publicación de estos contenidos, las redacciones están incorporando secciones de verificación (Alonso, 2017) e instrumentos fiables para detectar fake news (Vizoso y Vázquez-Herrero, 2019).

Las noticias falsas son una realidad que afecta a distintas generaciones. En relación con el sector poblacional que nos ocupa en este estudio, los estudiantes universitarios, una fórmula efectiva de frenar su propagación es fomentar el espíritu crítico de estos jóvenes. Para ello, es necesario adaptar la educación al entorno tecnológico actual (Rojas et al., 2018). En un informe elaborado por un grupo de expertos, a petición de la Comisión Europea (2018b), se insiste en la necesidad de promover la alfabetización mediática e informativa para neutralizar la desinformación y facilitar la libre navegación de los internautas. Por nuestra parte, consideramos que estas acciones formativas serán más efectivas y certeras si se diseñan sobre un diagnóstico previo. De manera que en la misma línea de investigaciones anteriores (Catalina-Grande, Sousa y Silva, 2019; Chandra, Surjandy y Ernawaty, 2017; Figueira y Santos, 2019), la intención con este trabajo es conocer la relación que mantienen los estudiantes de los grados en Comunicación y Comunicación Digital con las noticias falsas publicadas en la Red, de cara a trabajar con ellos esta temática en el aula. Máxime cuando hablamos de instruir y habilitar, desde la teoría y el manejo práctico de herramientas tecnológicas, a los futuros profesionales de la información.

\section{Metodología}

La investigación responde a un diseño mixto. Se lleva a cabo un doble estudio cuantitativo (análisis descriptivo y análisis de la varianza) y cualitativo (entrevista estructurada). En el caso de la parte cuantitativa, la metodología elegida ha sido descriptiva, siendo nuestro objetivo conocer el comportamiento y la percepción de los estudiantes universitarios acerca del fenómeno fake news, así como explorar vínculos asociando grupos de variables.

Para la obtención de los datos elaboramos un cuestionario que consta de dos bloques. El primero es un apartado donde se recogen las características identificativas del alumno mediante una serie de datos, personales y académicos, de los que se derivan las variables estudiadas: género (hombre o mujer); edad (18-20 años, 21-23 años, 24-28 años o más de 28 años); grado (Ciencias de la Comunicación o Comunicación Digital) y ciclo académico ( $1^{\circ}$ ciclo: $1^{\circ}$ y $2^{\circ}$ curso de la carrera o $2^{\circ}$ ciclo: $3^{\circ}$ y $4^{\circ}$ curso). El segundo bloque es una escala tipo Likert con 23 ítems, donde para cada pregunta (excepto la 5, que se debe responder con las alternativas Sí o No; y las preguntas 7 y 8 con respuesta múltiple) existen cuatro opciones de respuesta cuyos valores oscilan entre 1 y 4 puntos: 1 = nada de acuerdo (NA); 2 = poco de acuerdo (PA); 3 = bastante de acuerdo (BA); y $4=$ completamente de acuerdo (CA).

Por tratarse de un instrumento de elaboración propia, se llevaron a cabo unas pruebas que permitieran asegurar la validez del cuestionario. Para ello, se solicitó la colaboración de un grupo de expertos, formado por siete profesores universitarios del área de las Ciencias Sociales, quienes evaluaron el instrumento a partir de cuatro indicadores: la coherencia de las preguntas con los objetivos de la investigación; la claridad y la adecuación del lenguaje de estos ítems a la muestra; y su opinión acerca de transformar o eliminar algún ítem, o bien 
aportar alguna nueva pregunta al cuestionario. El feedback obtenido nos indicó los cambios a realizar en la primera versión de la escala.

Se ha trabajado con una población concreta y el cuestionario se aplicó de manera anónima a una muestra integrada por 118 alumnos matriculados en los grados en Ciencias de la Comunicación y Comunicación Digital del Centro Universitario San Isidoro, en Sevilla, adscrito a la Universidad Pablo de Olavide. Hemos empleado un muestreo dirigido y por conveniencia, que está enfocado a estos estudiantes, pues como profesorado es nuestra intención conocer sus relaciones con las fake news. De manera que este diagnóstico previo forma parte de la experiencia de trabajar en el aula con los alumnos de este Centro, permitiéndonos diseñar un material y unas actividades personalizadas en torno a las noticias falsas que se adapten y respondan a sus necesidades formativas, habilidades relacionadas con la identificación y la verificación de contenidos publicados en la Red, niveles de consumo y apreciaciones personales.

Entendemos que los resultados de la investigación sean válidos, pero no aplicables al amplio universo que conforman los estudiantes universitarios, pues trabajamos con un ecosistema docente delimitado. Sin embargo, sí serían extrapolables el propio estudio y el cuestionario diseñado, ya que pueden ser empleados por el profesorado como un paso previo y preparatorio antes de trabajar en sus clases la temática de las fake news. Pues cada vez son más frecuentes las experiencias y los proyectos docentes relacionados con la identificación y el tratamiento de este tipo de contenidos digitales (Auberry, 2018; Cebrián Robles, 2019; Herrero-Diz et al., 2019; Musgrove et al., 2018).

Por su parte, las pruebas para analizar los datos y el contraste de hipótesis se ejecutaron con el programa SPSS 22, y fueron: un estudio descriptivo, a nivel de puntuaciones medias (M) y desviaciones típicas (DT), y de porcentajes por ítems; y un análisis de la varianza, para averiguar si son significativas las diferencias obtenidas por los grupos estudiados en función de las variables. Tras comprobar que los resultados de la muestra no siguen una distribución normal, este análisis se realizó mediante la prueba de $U$ de Mann Whitney (para las variables dicotómicas) y la prueba de Kruskal Wallis (para las variables con más de dos grupos). Las pruebas devuelven un estadístico (p), de manera que cuando este es igual o menor de 0,05 la variable es significativa, por lo que rechazamos la hipótesis nula y afirmamos que existen diferencias entre los grupos de esa variable.

Para apoyar y complementar con información cualitativa los datos estadísticos derivados del estudio cuantitativo se empleó la técnica de la entrevista estructurada con preguntas abiertas. En concreto, este segundo cuestionario está compuesto por 6 ítems (Tabla 1) y

Tabla 1. Preguntas abiertas de la entrevista

\section{Preguntas}

1. ¿Qué procedimientos conoces para identificar una fake new?

2. ¿A qué temática pertenecen las fake news que te resultan más creíbles?

3. ¿Qué asignaturas del Grado en Ciencias de la Comunicación o Comunicación Digital relacionas con la identificación y la gestión de fake news?

4. ¿Qué organizaciones están detrás de la difusión masiva de fake news en Internet y con qué objetivos?

5. ¿Qué papel juegan los medios de comunicación en la distribución online de fake news?

6. ¿Qué consecuencias tienen las fake news en la ciudadanía?

Fuente: elaboración propia 
fueron entrevistados 12 universitarios. De esta manera, tal y como sugieren Cassany, Cortiñas y Elduque (2018: 12), esta doble metodología permite obtener datos cuantificables, y también «percibir y reportar las inquietudes, los matices y las sensaciones expresadas por los propios estudiantes». Se aplican dos formas de aproximación a la realidad estudiada, que no son consideradas excluyentes sino integradas en la misma investigación (Romero Tena, 1999).

\section{Análisis y resultados}

Para llevar a cabo el análisis, hemos categorizado el cuestionario en cuatro bloques temáticos o subescalas, cada una de las cuales está referida a un aspecto concreto del fenómeno fake news: las pautas de conducta y de consumo de los universitarios; la formación y el conocimiento de estos usuarios; las consecuencias y el arraigo social de estos contenidos; y los intereses y los organismos que existen detrás de la difusión de este tipo de noticias.

\subsection{Pautas de conducta y de consumo}

Respecto a la primera subescala: las pautas de conducta y de consumo de los estudiantes universitarios como prosumidores (Tabla 2), la mayoría de los sujetos entrevistados (79\%) percibe que no consume noticias falsas de manera periódica. Igualmente, una amplia mayoría ha elegido las opciones nada o poco de acuerdo y, por tanto, no comparte $(95,8 \%)$ y no da

Tabla 2. Pautas de conducta y de consumo

\begin{tabular}{|c|c|c|c|c|}
\hline Preguntas & 1 (NA) & 2 (PA) & 3 (BA) & 4 (CA) \\
\hline $\begin{array}{l}\text { Percibes que consumes noticias falsas de } \\
\text { manera periódica }\end{array}$ & $25,4 \%$ & $53,4 \%$ & $16,9 \%$ & $4,2 \%$ \\
\hline $\begin{array}{l}\text { Percibes que has leído fake news en las } \\
\text { últimas } 48 \text { horas }\end{array}$ & $33,1 \%$ & $28,8 \%$ & $22,9 \%$ & $15,3 \%$ \\
\hline Compartes noticias falsas en Internet & $79,7 \%$ & $16,1 \%$ & $2,5 \%$ & $1,7 \%$ \\
\hline Das like a noticias falsas & $66,9 \%$ & $25,4 \%$ & $5,9 \%$ & $1,7 \%$ \\
\hline \multirow[t]{2}{*}{$\begin{array}{l}\text { Denuncias en las redes sociales si detectas } \\
\text { que una información es falsa }\end{array}$} & $38,1 \%$ & $28 \%$ & $16,9 \%$ & $16,9 \%$ \\
\hline & Sí & No & & \\
\hline \multirow[t]{2}{*}{$\begin{array}{l}\text { Alguna vez he creado y difundido una } \\
\text { información falsa }\end{array}$} & $76,3 \%$ & $23,7 \%$ & & \\
\hline & Titulares & Fotos & Cuerpo & Vídeos \\
\hline $\begin{array}{l}\text { ¿A qué parte de la noticia le prestas más } \\
\text { atención? }\end{array}$ & $53 \%$ & $11,1 \%$ & $19,7 \%$ & $16,2 \%$ \\
\hline
\end{tabular}


like (92,4\%) a este tipo de contenidos. Pero aunque un $90 \%$ de los encuestados manifiesta que nunca ha creado o difundido una fake new, casi el $40 \%$ de estos jóvenes considera haber leído alguna en las últimas 48 horas.

Por otro lado, un $34 \%$ está bastante o completamente de acuerdo a la hora de afirmar que cuando detectan una información que no es verdadera lo comunican y lo denuncian en sus perfiles de redes sociales. Se aprecian diferencias significativas respecto al ciclo académico, de modo que este comportamiento es más frecuente en los estudiantes de $1^{\circ}$ ciclo (Media $=2,32$, Desviación Típica $=1,14$ ) (grado de significación del análisis de la varianza-p $=0,033)$ que en los de $2^{\circ}$ ciclo $(M=1,86, D T=0,98)$.

Por último, en relación con sus hábitos de lectura, para el 53,4\% de los futuros comunicadores, el titular es la parte de la noticia a la que prestan mayor atención, seguidas por este orden del cuerpo del artículo (19,5\%), los vídeos (16\%) y las fotografías (11\%). Así pues, podemos deducir que a pesar de tratarse de una generación eminentemente audiovisual, estos estudiantes del grado en Comunicación y Comunicación Digital se detienen en leer y juzgan la calidad y la credibilidad de las noticias en base a sus textos, y no sólo a través del material gráfico que las acompañan.

\subsection{Conocimiento y fORmación ACAdÉmica}

Un segundo bloque temático hace alusión a la importancia que tiene el conocimiento y la formación académica de los usuarios respecto a la manera en la que gestionan su relación con las noticias falsas publicadas en Internet (Tabla 3). En este sentido, la mayoría de los estudiantes $(73,7 \%)$ están bastante de acuerdo o completamente de acuerdo con el ítem «Sabes cómo contrastar una noticia publicada en Internet». Al estudiar las variables, se aprecian diferencias significativas en los resultados de esta pregunta al tener en cuenta el

Tabla 3. Conocimiento y formación académica

\begin{tabular}{|c|c|c|c|c|}
\hline Preguntas & 1 (NA) & 2 (PA) & 3 (BA) & 4 (CA) \\
\hline $\begin{array}{l}\text { Sabes cómo contrastar una noticia publicada en } \\
\text { Internet }\end{array}$ & $6,8 \%$ & $19,5 \%$ & $44,9 \%$ & $28,8 \%$ \\
\hline $\begin{array}{l}\text { Considero que la población, en general, distingue } \\
\text { una noticia falsa de otra verdadera }\end{array}$ & $43,2 \%$ & $51,7 \%$ & $4,2 \%$ & $0,8 \%$ \\
\hline Conoces el significado del término POSVERDAD & $16,1 \%$ & $20,3 \%$ & $22 \%$ & $41,5 \%$ \\
\hline Conoces el significado del término INFOXICACIÓN & $26,3 \%$ & $8,5 \%$ & $7,6 \%$ & $57,6 \%$ \\
\hline $\begin{array}{l}\text { En la escuela deberían advertir y formar sobre las } \\
\text { noticias falsas }\end{array}$ & $0 \%$ & $3,4 \%$ & $23,7 \%$ & $72,9 \%$ \\
\hline $\begin{array}{l}\text { La formación académica en Ciencias de la } \\
\text { Comunicación o Comunicación Digital contribuye a } \\
\text { evitar las noticias falsas }\end{array}$ & $0 \%$ & $11 \%$ & $38,1 \%$ & $50,8 \%$ \\
\hline
\end{tabular}

Fuente: elaboración propia 
género, la edad y el ciclo académico, pues el conocimiento al que nos referimos es superior en los grupos de hombres $(M=3,14$, DT =0,86) $(p=0,043)$; de los alumnos de mayor edad, a partir de 24 años, con una media que se aproxima a los 4 puntos, que es el valor máximo de la escala $(p=0,005)$; $y$ de aquellos que cursan el $2^{\circ}$ ciclo de la carrera $(M=3,28, D T=0,72)$ $(p=0,001)$.

Al profundizar en los procedimientos empleados, las entrevistas nos han permitido conocer que son: contrastar la información con otras fuentes en otros medios de comunicación o en otras plataformas, acudir a la fuente oficial o estudiar quién firma la noticia (A7, que se refiere al Alumno número 7 ). Y también:

- «Verificar que la URL sea segura, y que las fechas de todas las noticias coincidan» (A1).

- «Con herramientas como Snopes» (A2).

- «Acudir a redes sociales que desmienten estas noticias» (A5).

La mayoría de los alumnos afirman que conocen el significado de los términos «posverdad» $(63,56 \%)$ e «infoxicación» $(65,26 \%)$. Respecto a estos ítems, se han encontrado diferencias significativas en función de las variables edad y ciclo académico, pues las respuestas afirmativas predominan entre los estudiantes que cursan el $2^{\circ}$ ciclo [Posverdad $(M=3,14$, $\mathrm{DT}=1,06)(p=0,034)$ / Infoxicación $(M=3,86, D T=0,53)(p=0)]$, y entre los de mayor edad, con más de 23 años, donde la media alcanza el 4 , que es la puntuación máxima $(p=0)$. Se trataría de quienes han avanzado más en sus estudios. Por consiguiente, estos universitarios deben tener un mayor conocimiento y presentar mejores destrezas en relación con el panorama mediático y la gestión de contenidos digitales.

Por su parte, el $88,99 \%$ de los estudiantes están bastante o completamente de acuerdo con el hecho de que la formación académica en Ciencias de la Comunicación o Comunicación Digital contribuye a evitar las fake news. Respecto a las asignaturas que más se relacionan con la identificación y la gestión de noticias falsas, durante las entrevistas han manifestado que son Identidad y Reputación Online, y Gestión de Plataformas de Contenidos, en el caso del Grado en Comunicación Digital; Redacción Periodística, Periodismo Digital y las Teorías de la Comunicación y la Información en el Grado en Ciencias de la Comunicación; y materias comunes en ambas carreras como Derecho y Documentación.

Solo el $5 \%$ de los alumnos percibe que la población, en general, distingue una noticia falsa de otra verdadera. Existen diferencias significativas al tener en cuenta la variable ciclo académico, siendo los estudiantes que cursan asignaturas del $1^{\circ}$ ciclo los que peor han valorado este ítem $(M=1,51, D T=0,56)(p=0,025)$. Y este dato explicaría porqué prácticamente todos los estudiantes encuestados $(96,61 \%)$ están de acuerdo con que ya desde la escuela se debería advertir y formar a los menores sobre las noticias falsas.

\subsection{Consecuencias y arraigo social}

Respecto a los efectos sociales de las noticias falsas (Tabla 4), la mayoría de los futuros comunicadores ( $89 \%$ ) considera que no son inofensivas. Mientras un $25 \%$ sí está de acuerdo con que son divertidas, detectándose en las respuestas a este ítem diferencias respecto a la 
Tabla 4. Consecuencias y arraigo social

\begin{tabular}{|l|c|c|c|c|}
\hline \multicolumn{1}{|c|}{ Preguntas } & $\mathbf{1}$ (NA) & $\mathbf{2}$ (PA) & $\mathbf{3}$ (BA) & $\mathbf{4}$ (CA) \\
\hline Las noticias falsas son inofensivas & $71,2 \%$ & $17,8 \%$ & $6,8 \%$ & $4,2 \%$ \\
\hline Las noticias falsas son divertidas & $34,7 \%$ & $40,7 \%$ & $22,9 \%$ & $1,7 \%$ \\
\hline $\begin{array}{l}\text { La sociedad prefiere noticias afines a sus } \\
\text { intereses que la propia verdad }\end{array}$ & $9,3 \%$ & $11 \%$ & $50 \%$ & $29,7 \%$ \\
\hline Las fake news son una moda pasajera & $55,9 \%$ & $30,5 \%$ & $11 \%$ & $2,5 \%$ \\
\hline Las noticias falsas son inofensivas & $71,2 \%$ & $17,8 \%$ & $6,8 \%$ & $4,2 \%$ \\
\hline
\end{tabular}

Fuente: elaboración propia

variable género en favor de los hombres, cuya valoración es más positiva ( $M=2,1, D T=0,84)$ $(p=0,036)$ que la de sus compañeras $(M=1,78, D T=0,74)$. Por su parte, el $86,4 \%$ considera que la publicación de estos contenidos no responde a una moda pasajera.

$Y$ respecto a las causas de su consumo, casi el $80 \%$ de estos jóvenes considera que la sociedad prefiere noticias afines a sus intereses que la propia verdad. Al estudiar las respuestas según la edad, se encuentran diferencias significativas, siendo los estudiantes con una edad comprendida entre los 24 y los 28 años $(M=3,20$, $D T=0,40)(p=0,03)$ quienes están más de acuerdo con esta afirmación.

El consumo de fake news tiene unas consecuencias en la ciudadanía que, según han desvelado los estudiantes en las entrevistas, son desinformación, difusión de ideas erróneas, falsa percepción de la realidad, engaño, confusión, manipulación de la opinión pública, alarma social, "seguimiento de corrientes peligrosas como el movimiento antivacunas» (A1), y «establecimiento de una "pseudocracia"» (A12).

\subsection{INTERESES Y ORGANISMOS IMPLICADOS}

Esta categoría recoge aquellos ítems relacionados con los intereses y los organismos que promueven la publicación masiva de noticias falsas en la Red (Tabla 5). Y en este sentido, la mayoría de los estudiantes están bastante o completamente de acuerdo con el hecho de que estos contenidos responden a intereses políticos (72\%) y económicos (67\%). En este caso, las diferencias significativas se aprecian en la variable género y a favor de los hombres, quienes le han otorgado a estos ítems [Intereses Políticos $(M=3,22, D T=0,74)$ $(p=0,004) /$ Intereses Económicos $(M=3,08, D T=0,8)(p=0,012)]$ una mayor puntuación que las mujeres. En cualquier caso, estos datos se correlacionan con la creencia, anteriormente comentada, de que estas informaciones no son inocuas ni inofensivas.

Los resultados obtenidos en este bloque se refuerzan con las respuestas a las preguntas abiertas de la entrevista, donde los futuros comunicadores han manifestado que las organizaciones que en mayor medida están detrás de la difusión masiva de noticias falsas en Internet son los medios de comunicación, los partidos políticos y los gobiernos. E incluso «cualquiera para obtener un beneficio o atacar la reputación digital de otra persona» (A2). Y que algunos de los objetivos de este proceder son: 
Tabla 5. Intereses y organismos implicados

\begin{tabular}{|c|c|c|c|c|}
\hline Preguntas & 1 (NA) & 2 (PA) & 3 (BA) & 4 (CA) \\
\hline Las noticias falsas responden a intereses políticos & $1,7 \%$ & $26,3 \%$ & $44,9 \%$ & $27,1 \%$ \\
\hline $\begin{array}{l}\text { Las noticias falsas responden a intereses } \\
\text { económicos }\end{array}$ & $4,2 \%$ & $28,8 \%$ & $44,1 \%$ & $22,9 \%$ \\
\hline $\begin{array}{l}\text { A las redes sociales les interesa publicar noticias } \\
\text { falsas }\end{array}$ & $3,4 \%$ & $32,2 \%$ & $37,3 \%$ & $27,1 \%$ \\
\hline $\begin{array}{l}\text { Los medios de comunicación contribuyen a la } \\
\text { difusión de fake news }\end{array}$ & $9,3 \%$ & $29,7 \%$ & $40,7 \%$ & $20,3 \%$ \\
\hline \multirow[t]{2}{*}{$\begin{array}{l}\text { Facebook quiere erradicar las fake news de sus } \\
\text { contenidos }\end{array}$} & $12,7 \%$ & $38,1 \%$ & $27,1 \%$ & $22 \%$ \\
\hline & RRSS & Medios & Blogs & $\begin{array}{c}\text { Familia } \\
\text { y amigos }\end{array}$ \\
\hline ¿Qué fuente de información consideras más fiable? & $2,6 \%$ & $85,5 \%$ & $3,4 \%$ & $8,5 \%$ \\
\hline
\end{tabular}

Fuente: elaboración propia.

- «Confundir a la sociedad aprovechando la infoxicación e inducir a la opinión pública hacia ideas erróneas» (A4).

- «Con intención satírica para hacer reír» (A6). Y pone como ejemplo la publicación El Mundo Today.

- «Ocultar lo que ocurre en partidos políticos y acusar con ellas a los otros» (A9).

Para estos universitarios, la fuente informativa que consideran más fiable son los medios de comunicación (85,6\%), seguida, a mucha distancia, de su propia familia y los amigos $(8,5 \%)$, los blogs $(3,4 \%)$ y, por último, las redes sociales $(2,5 \%)$. Y en relación con las temáticas que les resultan más creíbles, en las entrevistas manifiestan que son, principalmente, la solidaridad «porque poseen un factor sentimental» (A10), deporte y «aquellas en las que intervenga un cuerpo de seguridad como la policía o los bomberos» (A2).

Respecto a las plataformas online que dan cobertura a las noticias falsas, según el sumatorio de las respuestas afirmativas, los alumnos consideran que son, en mayor medida, las redes sociales $(64,4 \%)$, seguidas de los medios de comunicación $(61 \%)$. Precisamente, sobre el papel que desempeñan los mass media en la distribución online de fake news, las entrevistas revelan percepciones enfrentadas:

- «Es vital, tanto para desmentir como para darles más viralidad» (A1).

- «Funcionan como un altavoz que lanza este tipo de noticias» (A2).

- «Usan los títulos clickbaits para jugar con la posverdad» (A12).

En los dos extremos, ayudan a su divulgación o las contrastan, pues

- «Tienen medios para reconocerlas» (A3).

- «Deben ser un filtro, incluso proveer a los lectores de armas para detectarlas» (A7). 
Cabe destacar que la mitad de los encuestados creen que Facebook no quiere erradicar las fake news de su muro; a pesar de que la compañía ha anunciado la puesta en marcha de herramientas propias para frenar la difusión de estos contenidos, así como otros procedimientos tales como limitar el número de veces que se pueden reenviar los mensajes para evitar la difusión de bulos, o la instalación de programas de verificación de datos que no la eliminan, pero que pueden marcar una noticia como falsa (Facebook, 2019). En esta ocasión, las diferencias significativas se detectan en la variable edad, y son los alumnos que tienen entre 21 y 23 años los que menos confían en las intenciones de esta red social $(M=2,43$, DT $=0,91)(p=0,046)$.

\section{Discusión/Conclusiones}

Las noticias falsas se configuran como un tema actual, con una amplia presencia en el debate público por el papel que desempeñan en el control social tanto desde el campo de la política como en la esfera mediática. Por ello, este fenómeno debe investigarse en el ámbito de la comunicación y no obviarse desde un punto de vista educativo. En nuestro caso, el propósito ha sido indagar en las opiniones y actitudes de los estudiantes universitarios de Comunicación y Comunicación Digital ante las fake news. A través de un cuestionario y una entrevista aplicados a una muestra de conveniencia, el estudio proporciona información sobre diversos parámetros: conducta y consumo, formación académica, procedimientos de verificación, consecuencias sociales e intereses. Y los resultados evidencian que los alumnos consultados no son ajenos a estos contenidos y son capaces de manifestar su propia opinión al respecto.

Respecto a las pautas de conducta de los estudiantes como prosumidores, la mayoría percibe que no lee noticias falsas de manera periódica, y que tampoco las crea o las comparte. Es más, un $34 \%$ de los participantes, especialmente en el grupo de los alumnos del $1^{\circ}$ ciclo, indica que si detecta una fake new advierte y denuncia su presencia en sus propias redes sociales.

Desde un punto de vista académico, el $90 \%$ de los encuestados considera que su formación específica en Ciencias de la Comunicación o Comunicación Digital favorece la capacidad de determinar la veracidad de una noticia publicada en Internet. De hecho, son capaces de citar técnicas de verificación y herramientas fact-checking. Igualmente, la mayoría de estos universitarios, y en mayor medida aquellos que cursan el segundo ciclo de la carrera, conocen el significado de los términos «posverdad» e «infoxicación» ligados a la corriente fake news, pues se trabaja en torno a ellos en asignaturas varias como Identidad y Reputación Online, Gestión de Plataformas de Contenidos, Periodismo Digital o Documentación.

En un ámbito más general, estos universitarios perciben que la ciudadanía tiene problemas para distinguir una noticia real de otra falsa. Este dato coincide con el I Estudio sobre el impacto de las fake news en España (Europa Press, 2017), donde se confirma que un $86 \%$ de los españoles posee ciertas dificultades a la hora de establecer la veracidad de determinadas informaciones. Y en consecuencia, defienden la puesta en marcha de programas de alfabetización mediática y digital que deben comenzar desde la escuela (Bonilla del Río y Aguaded, 2018; Martín-Herrera, 2017; Rodríguez-de-Dios e Igartua, 2018). 
En relación con los efectos sociales de las fake news, casi todos los estudiantes opinan que estas noticias no son inofensivas. De hecho, para los futuros comunicadores profesionales, preferentemente en el caso de los hombres, la mayoría de estos contenidos responden, por este orden, a intereses políticos y económicos. Y piensan que los organismos que están detrás de su difusión son los medios de comunicación y los gobiernos para «ocultar lo que ocurre en los partidos políticos y confundir a la sociedad con ideas erróneas». También consideran que las fake news no responden a una moda pasajera, y que parte de su éxito se debe a que la sociedad prefiere leer noticias afines a sus intereses, aunque no sean verdaderas.

Los medios de comunicación son considerados por estos universitarios como la fuente informativa más fiable. Y sobre la vinculación de los mass media con las noticias falsas manifiestan opiniones variadas. Así pues, por un lado, creen que desempeñan un papel vital para actuar como filtro y desmentir estos mensajes; y por otro lado, los perciben como un altavoz que contribuye a su difusión.

Otro de los objetivos de la investigación era determinar variables que influyeran en las percepciones de los universitarios sobre las noticias falsas. En base a las pruebas de contraste, concluimos que las variables edad y ciclo académico son las que manifiestan una relación estadísticamente significativa con un mayor número de preguntas. Se advierte que los alumnos de mayor edad y que cursan los últimos años de la carrera se perciben a sí mismos como más instruidos y competentes para gestionar las noticias falsas en internet. Interpretamos que este resultado se corresponde con la formación recibida, las habilidades adquiridas y el grado de madurez esperable en estos grupos. Por el contrario, no se aprecian diferencias significativas en ningún ítem al atender a los grupos de la especialidad académica: Ciencias de la Comunicación y Comunicación Digital.

El diagnóstico realizado con este estudio nos sirve para plantear conclusiones a nivel docente. En este sentido, parece necesario afianzar la presencia de cuestiones relativas a las noticias falsas y los procesos de desinformación en los temarios de aquellas asignaturas de los Grados en Comunicación y Comunicación Digital que son más afines a estas materias. Igualmente, se plantea el diseño e impartición de talleres y seminarios específicos, desarrollados por profesores y profesionales, y dirigidos a estos estudiantes universitarios. En ambos casos, estas iniciativas educativas deben estar enfocadas a lograr las siguientes habilidades comunicativas: detectar noticias falsas: dónde se originan y por dónde circulan, con el fin de evitar su impacto negativo a la hora de informarse o investigar; comprobar la veracidad de las fuentes; y conocer los intereses y organismos presentes tras su difusión a través de la Red. La intención es, por un lado, formarlos como ciudadanos adultos capaces de gestionar con criterio su consumo mediático en la Red; y por otro, prepararlos de un modo más específico para convertirse en profesionales que tendrán que identificar, verificar, filtrar, desmentir... estos mensajes en el desempeño de sus tareas laborales, con la responsabilidad y la labor social que ello implica.

Por último, teniendo en cuenta los continuos cambios que experimentan los fenómenos ligados a Internet, dentro del marco de las implicaciones, se proponen revisiones periódicas de la investigación que permitan conocer cómo evolucionan en el tiempo las actitudes de los estudiantes universitarios de Ciencias de la Comunicación ante las noticias falsas publicadas en la Red. 


\section{Bibliografía}

AGUADED, Ignacio (2014). «Desde la infoxicación al derecho a la comunicación». Comunicar, 42, 7-8. DOI: $10.3916 / C 42-2014-a 1$

ALONSO, Patricia (2017). «Fake news y posverdad en tiempos de populismos: lecciones para periodistas». Cuadernos de Periodistas, 34, 77-84.

AUBERRY, Kendra (2018). «Increasing students' ability to identify fake news through information literacy education and content management systems». The Reference Librarian, 59(4), 179-187. DOI: 10.1080/02763877.2018.1489935

BLANCO-HERRERO, David y ARCILA-CALDERÓN, Carlos (2019). «Deontología y noticias falsas: estudio de las percepciones de periodistas españoles». El profesional de la información, 28(3). DOI: 10.3145/epi.2019.may.08

BONILLA-DEL-RÍO, Mónica y AGUADED, Ignacio (2018). «La escuela en la era digital: Smartphones, APPS y programación en Educación Primaria y su repercusión en la competencia mediática del alumnado». Pixel-Bit: Revista de Medios y Educación, 53, 151-163. DOI: 10.12795/pixelbit.2018. i53.10

CASSANY, Roger, CORTIÑAS, Sergi y ELDUQUE, Albert (2018). "Comunicar la ciencia: El perfil del periodista científico en España». Comunicar, 26(55), 9-18. DOI: 10.3916/C55-2018-01

CATALINA-GRANDE, Beatriz, SOUSA, Jorge Pedro y SILVA, L. C. (2019). "Consumo de noticias y percepción de fake news entre estudiantes de Comunicación de Brasil, España y Portugal». Revista de Comunicación, 18(2), 93-115. DOI: 10.26441/RC18.2-2019-A5

CEBRIÁN ROBLES, Daniel (2019). «Identificación de noticias falsas sobre ciencia y tecnología por estudiantes del grado de Primaria». Píxel-Bit. Revista de Medios y Educación, 55, 23-36. DOI: 0.12795/pixelbit.2019.i55.02

CHANDRA, Yakob Utama, SURJANDY y ERNAWATY (2017). «Higher education student behaviors in spreading fake news on social media: A case of LINE group». In International Conference on Information Management and Technology (ICIMTech). IEEE, págs. 54-59. DOI: 10.1109/ ICIMTech.2017.8273511

COMISIÓN EUROPEA (2018a). La lucha contra la desinformación en línea: un enfoque europeo. COM (2018) 236 final. Recuperado de https://bit.ly/2IZb8KX

COMISIÓN EUROPEA (2018b). A multi-dimensional approach to disinformation: Final report of the high level expert group on fake news and online disinformation. Luxembourg: European Union. Recuperado de https://goo.gl/bk9NYg

EUROPA PRESS (2017). «El $86 \%$ de los españoles tiene dificultades para distinguir entre "fake news" y noticias verdaderas, según un estudio». Europa Press, 31 de mayo. Recuperado de https://bit. Iy/2SJyLKK

FACEBOOK (2019). «Facebook lanza su programa de verificación de datos externo en España». Facebook. Newsroom, 12 de marzo. Recuperado de https://bit.ly/2UwAMul

FERNÁNDEZ-GARCÍA, Nuria (2017). «Fake news: una oportunidad para la alfabetización mediática». Nueva Sociedad, 269, 66-77.

FIGUEIRA, João y SANTOS, Silvio (2019). «La percepción de las noticias falsas en universitarios de Portugal: análisis de su consumo y actitudes». El profesional de la información, 28(3), e280315. DOI: 0.3145/epi.2019.may.15 
GRANDE, Víctor (2018). Infoxicación, la nueva ignorancia / \& entrevista. Youtube. Recuperado de https:// bit.ly/2E8BE2W

HERNÁNDEZ-SERRANO, María-José, RENÉS-ARELLANO, Paula, GRAHAM, Gary y GREENHILL, Anita (2017). «Del prosumidor al prodiseñador: el consumo participativo de noticias». Comunicar, 50, 77-88. DOI: 10.3916/C50-2017-07

HERRERO-DIZ, Paula, CONDE-JIMÉNEZ, Jesús, TAPIA-FRADE, Alejandro y VARONA-ARAMBURU, David (2019). "The credibility of online news: an evaluation of the information by university students». Culture and Education, 31(2), 407-435. DOI: 10.1080/11356405.2019.1601937

HOROWITZ, Minna A. (2019). «Disinformation as warfare in the digital age: dimensions, dilemmas, and solutions». Journal of Vincentian Social Action, 4(2).

LEVIT, Jorge (2017). «La "posverdad" argentina». La Capital, 6 de septiembre. Recuperado de https:// bit.ly/2YbHLKz

LÓPEZ-BORRULL, Alexandre, VIVES-GRÀCIA, Josep y BADELL, Joan-Isidre (2018). «Fake news, ¿amenaza u oportunidad para los profesionales de la información y la documentación?». El profesional de la información, 27(6), 1346-1356. DOI: 10.3145/epi.2018.nov.17

MAGALLÓN-ROSA, Raúl (2017). «La biblioteca digital sobre Donald Trump. Fact-checking frente a fake news». Estudios sobre el Mensaje Periodístico, 24(1), 273-282. DOI: 10.5209/ESMP.59949

MARTENS, Bertín, AGUIAR, Luis, GÓMEZ-HERRERA, Estrella y MUELLER-LANGER, Frank (2018). The digital transformation of news media and the rise of disinformation and fake news. Digital economy working paper 2018-02. Joint Research Centre Technical Reports. DOI: 10.2139/ssrn.3164170

MARTÍN-HERRERA, Inmaculada (2017). «Una alfabetización múltiple con la prensa escolar digital. Propuesta de un modelo referencial». Razón y Palabra, 21(3_98), 99-117.

MCNAIR, Brian (2018). Fake news: Falsehood, fabrication and fantasy in journalism. London: Routledge. ISBN: 9781138306790

MOLINA-CAÑABATE, Juan Pedro (2019). «Unfaking news. Como combatir la desinformación». Estudios sobre el Mensaje Periodístico, 25(2), 1267-1269. DOI: 10.5209/esmp.64838

MUSGROVE, Ann T., POWERS, Jillian R., REBAR, Lauri C. y MUSGROVE, Glenn J. (2018). «Real or fake? Resources for teaching college students how to identify fake news". College \& Undergraduate Libraries, 25(3), 243-260. DOI: 10.1080/10691316.2018.1480444

RODRÍGUEZ-DE-DIOS, Isabel e IGARTUA, Juan-José (2018). «Skills of digital literacy to address the risks of interactive communication». In Information Resources Management Association (Ed.). Information and technology literacy: Concepts, methodologies, tools, and applications. Hershey, PA: IGI Global, págs. 621-632. DOI: 10.4018/978-1-5225-3417-4.ch034

ROJAS, David, FERNÁNDEZ, Pedro, RODRÍGUEZ, Mauricio y GUILLÉN, Alberto (2018). «Plataforma de entrenamiento para detectar fake news en los Recursos Educativos como Internet». Enseñanza y Aprendizaje de Ingeniería de Computadores, 8.

ROMERO TENA, Rosalía (1999). La integración de las nuevas tecnologías los grupos de trabajo de la provincia de Huelva. Tesis Doctoral. Universidad de Sevilla.

TANDOC, Edson C., LIM, Zheng Wei y LING, Richard (2017). «Defining fake news. A typology of scholarly definitions». Digital journalism. 6(2), 137-153. DOI: 10.1080/21670811.2017.1360143

VIZOSO, Ángel y VÁZQUEZ-HERRERO, Jorge (2019). «Plataformas de fact-checking en español. Características, organización y método». Communication \& Society, 32(1), 127-144. DOI: 10.15581/003.32.1.127-144

VOSOUGHI, Soroush, ROY, Deb y ARAL, Siman (2018). "The spread of true and false news online». Science, 359 (6380), 1146-1151. 